DIW BERLIN

Discussion Papers
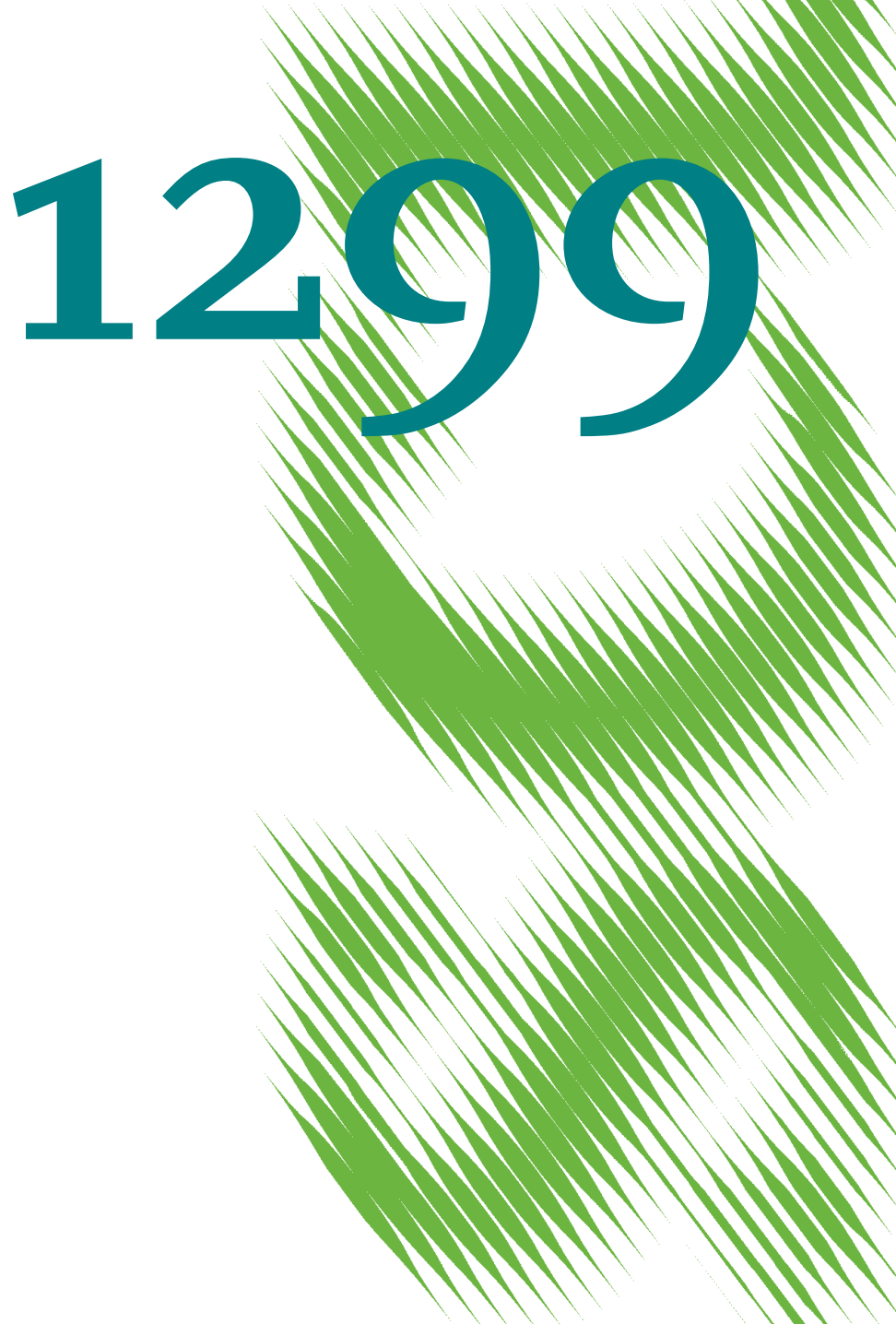

European Electricity Grid Infrastructure Expansion in a 2050 Context 
Opinions expressed in this paper are those of the author(s) and do not necessarily reflect views of the institute.

IMPRESSUM

(C) DIW Berlin, 2013

DIW Berlin

German Institute for Economic Research

Mohrenstr. 58

10117 Berlin

Tel. $+49(30) 89789-0$

Fax +49 (30) $89789-200$

http://www.diw.de

ISSN print edition $1433-0210$

ISSN electronic edition 1619-4535

Papers can be downloaded free of charge from the DIW Berlin website:

http://www.diw.de/discussionpapers

Discussion Papers of DIW Berlin are indexed in RePEc and SSRN:

http://ideas.repec.org/s/diw/diwwpp.html

http://www.ssrn.com/link/DIW-Berlin-German-Inst-Econ-Res.html 


\title{
European Electricity Grid Infrastructure Expansion in a 2050 Context
}

\author{
Jonas Egerer* $\stackrel{* \dagger}{*}$ Clemens Gerbaulet ${ }^{\dagger \dagger}$, and Casimir Lorenz
}

May 2013

\begin{abstract}
The European climate targets until 2050 require an adaptation of the generation portfolio in terms of renewable and fossil based generation. Assumptions on the timeline of the targets and the availability and costs of generation technologies are used in energy system models to optimize the cost minimal system transformation. The results include investments in generation technologies and their national allocation. Yet, the models are limited to the national aggregation and lack the spatial resolution required to represent individual network investments and related costs. In this paper, we analyze the impact the results of an energy system model have on demand for network expansion in the European power grid in a line-sharp representation. A cost minimizing mixed-integer problem (MIP) model calculates where in the European electricity grid expansion needs to take place for different time steps (2020/30/40/50) in order to obtain minimal total costs for power plant dispatch and grid expansion. Scenarios based on the generation infrastructure options from the PRIMES EU-wide energy model scenarios invoke different expansion needs and are compared. The model allows investments in the AC network and an overlay DC grid. Resulting investment costs are compared to the numbers of the European Energy Roadmap 2050.
\end{abstract}

JEL Codes: C61, H54, L94

Keywords: Electricity, European Transmission Network, Investment Model

\footnotetext{
*Corresponding Author: jegerer@diw.de

${ }^{\dagger}$ German Institute for Economic Research (DIW Berlin), Department of Energy, Transportation, Environment, Mohrenstraße 58, 10117 Berlin.

${ }^{\ddagger}$ Berlin University of Technology, Workgroup for Infrastructure Policy (WIP), Straße des 17. Juni 135, 10623 Berlin.
} 


\section{Introduction}

The decarbonization of the electricity sector is a fundamental cornerstone in the European climate policy. Yet, the pathway for the transformation process is less clear regarding its timeline and the coordination of national and European actions. Additional uncertainty results from the availability of various technologies for low carbon generation (renewable technologies, nuclear, and carbon capture and storage - CCS) and their related costs. Adding to the uncertainty in system transformation, the design of the future European electricity grid is another topic of controversy. At the national and the European level, the institutional framework for grid planning has been somewhat standardized with the ten-year-network-development plan (TYNDP) procedures. Yet, starting from today's European transmission network, uncertainty for network development remains with respect to technology (e.g. AC vs. DC networks) as well as to the degree of cross-border system integration.

The TYNDP represents the most up to date reference for the upcoming transmission investments. This European plan is supposed to be compatible with the national investment plans and the guidelines for trans-European energy networks $(\overline{E C}, 2006)$. It identifies bottlenecks within Europe with regard to market integration issues, generation development, and security of supply. Within the next ten years, investment cost of $€ 104$ bn are estimated that go along with a forecasted reduction in generation cost of 5\%. (ENTSO-E, 2012a)

Furthermore, various studies assess investments in the European transmission network, partly along with investments in generation, until 2030 or 2050. The Roadmap 2050 report (EC, 2011) outlines possible ways to reach the $80 \%$ greenhouse-gas (GHG) reduction targets by 2050. Dependent on the scenario assumptions, significant investments in additional capacity of transmission lines are required. They result in transmission investment cost between $€ 30$ bn to $€ 93$ bn until 2050 .

This paper provides a bottom-up model based analysis of the European high-voltage electricity infrastructure development for the time horizon until 2050. It examines national scenario results of the PRIMES model (Capros, 1998) for three different scenarios with regard to optimal network investments. The remainder of this paper is structured as follows: the section 2 outlines the background of the applied model. Section 3 describes implementation of the model. The data and scenarios are presented in section 4 . Section 5 discusses the modeling results. In section 6 we draw conclusions. 


\section{The Infrastructure Investment Model}

The model application in this paper is based on the techno-economic ELectricity MODel (ELMOD) developed at the Technische Universität Dresden (Chair of Energy Economics), the Technische Universität Berlin (Workgroup for Infrastructure Policy) and the German Institute of Economic Research Berlin (Department of Energy, Transportation, Environment). "ELMOD is a large-scale spatial model of the European electricity market including both generation and the physical transmission network (DC Load Flow Approach)" (Leuthold et al., 2012). The code of the model is written in GAMS (General Algebraic Modeling System) either as an optimization or as a partial equilibrium problem and has been adjusted for various research questions. 1

Within the Energy Modelling Framework 28 (EMF28), we apply the model for an infrastructure assessment of the European electricity transmission system until 2050. The results provide insights in regional investment needs of national and cross-border lines, total cost and the distribution of cost on the European nations for each scenario. The EMF28 is a model comparison exercise for the long term development of the European energy sector. The scenarios elaborate the effects of technology choices and the implementation of climate policy targets focusing on the EU's climate targets for 2020 and 2050.

The EMF28 model comparison has been conducted with top-down energy system and general equilibrium models. Although these types of models allow for consideration of important inter-temporal variables of system development, they reduce the electricity infrastructure to a very simplistic level. Electricity exchange between two neighboring countries is included but only a transport model with directed flows is used. This approach has the following main shortcomings:

- The electricity load flow in a meshed network follows physical flow constraints resulting in loop-flows. This specific characteristic of electricity flows is not considered in transport models with directed flows;

- Linear cost curves for exchange capacity between two countries assume an incremental character of investments in line capacity. This neglects the lumpy character

\footnotetext{
${ }^{1}$ Examples are: For market design Weigt et al. (2010), for strategic market behavior Weigt and Hirschhausen (2008), for uncertainty and stochastic effects Abrell and Kunz (2012), for welfare distribution Egerer et al. (2012), for regulatory challenges Rosellón and Weigt (2011); Schill et al. (2011), and for energy system planning with the integration of renewable generation and transmission investment Leuthold et al. (2009); Egerer et al. (2009).
} 
of transmission investment;

- The national aggregation to one node per country is unable to capture national infrastructure needs and related cost. Yet large shares of the transmission network investments are motivated by unevenly distributed generation and demand within one country: $\left.\right|^{2}$

The bottom-up model that is applied in this analysis increases the level of detail on electricity infrastructure compared to top-down energy system and general equilibrium models. Thereby, it provides a better understanding of the network requirements for the scenario results of the top-down models. The fundamental elements in the bottom-up approach are the line-sharp resolution of the high-voltage network with a nodal energy balance, and the DC Load Flow (DCLF) implementation 3 It is therefore a useful tool to address the question of transmission investment from a techno-economic perspective. The following aspects are considered:

- The transmission system consists of four non-synchronized AC networks and several DC point-to-point connectors. The AC networks are modeled with the methodology of DC load flow. DC connections are modeled as directed flows to constrain electricity flows in a meshed system in accordance to physical line characteristics;

- Discrete investment decisions in transmission lines are enforced using a mixed integer model formulation with binary and integer variables;

- The investment choices include voltage upgrades to $380 \mathrm{kV}$ and additional circuits for AC lines. Additionally the model can invest in a set of point-to-point DC lines. Line specific investment costs provide a more realistic evaluation of the options and result in a deeper understanding for the scenario-specific infrastructure cost;

- The change of flow patterns by line upgrades or investment in new lines are considered in the optimization of the network topology.

\footnotetext{
${ }^{2}$ Examples are local restrictions for hydropower, reliance of conventional plants on cooling water, and proximity of coal power plants to harbors to access cheap import coal. Additional renewable generation capacities will further increase the average distance between generators and demand within one country.

${ }^{3}$ The DCLF simplification considers loop-flows in the transmission system as the flow pattern obliges to physical line characteristics (Schweppe, 1988). Yet, certain aspects of AC current, e.g. reactive power are neglected.
} 
Although the grid representation is far more detailed than in top-down models, several abstractions from reality remain in this approach, mainly due to restrictions in today's computation resources. The optimization is conducted separately for every decade in a consecutive order. The grid expansion result of one decade determines the starting topology for the next one. The options for expansion are limited to existing network links in the grid. The model allows voltage upgrade of lines with less than $380 \mathrm{kV}$ to $380 \mathrm{kV}$ and expansion of all existing AC lines of the European high voltage network. In addition the model can determine investments in an exogenously predefined DC overlay grid. Several other technical constraints (reactive power requirements, etc.) are excluded. N-1 security is considered by implementing a $20 \%$ transmission reliability margin. Expansion costs for investment in new lines are constant factors per kilometer and do not consider regional characteristics. The supply side of electricity is reduced from a block-sharp representation to an aggregated representation with different generation technologies per network node. The time resolution per model run is a set of 18 specific hours without constraints linking them. Thus, seasons, different demand levels, and availabilities of renewable generation are regarded, but no storage nor demand side management (DSM) technology is included.

One challenge of energy system planning lies in the interdependence of generation and transmission. In order to achieve the cost optimal energy system, a combined analysis of spatial generation and transmission investment is required. But due to the unbundling in generation and transmission companies, combined planning is currently not taking place on a national level. This analysis assumes that integrated system planning is limited to the top-down energy system models with their simplified network representation. Using their data on generation capacity, the transmission network is optimized ex-post without its more detailed costs being considered in the integrated planning.

Another aspect is the European coordination in planning and investing in the transmission network. The TYNDP (ENTSO-E, 2012a) provides some common planning ground on the European level. With the inter-TSO compensation mechanism and the lately discussed projects of common interest instruments for multilateral network development are at hand. On the other hand, national regulators and parliaments decide on national transmission plans and national strategies for renewable targets. This shows that transmission planning still mainly serves national purposes.

The single objective of European cost minimization is a reasonable approach for the level of detail and the European scale of the model. Thus, individual national interest 
are neglected $4^{4}$ The assessment is conducted with scenario runs on different levels of investment costs for cross-border lines. This evaluation does not consider national interest in market results and rent shifting. It reflects on the current scheme of national planning which is done on national level and imposes additional costs for multinational coordination.

All in all, the approach evaluates the infrastructure development and related cost for different EMF28 scenarios on a European scale. Thereby, it provides additional value by its line sharp resolution, various investment options, and consideration of different load and renewable levels.

\section{Model Implementation}

The investment model is formulated as a Mixed Integer Linear Problem (MILP). It contains two decision levels, the transmission investment and the market dispatch. The two stages are reduced to one level assuming perfect competition and a European central planner that expands the transmission network with the objective to minimize total system costs. The AC network investments cause a bi-linearity in the flow constraint which usually requires a non-linear problem (MINLP). To remain in a linear model world the model is solved iteratively with endogenous investment in transmission capacity. Yet, physical line characteristics are adjusted ex-post after each solve. The optimization problem is shown in (1) to (11).

$$
\begin{aligned}
\min \cos t= & \sum_{n, s, t}\left(g_{n, s, t} * M C_{n, s}\right) \\
& +\sum_{d}\left(\operatorname{expdc}_{d} * C d c_{d}\right) \\
& +\sum_{l}\left(u p_{l} * C u p_{l}+\exp _{l} * C \exp _{l}\right)
\end{aligned}
$$

\footnotetext{
${ }^{4}$ To use a multiobjective approach an MPEC or EPEC model would be required.
} 
s.t.

$$
\begin{aligned}
& 0=\sum_{s} g_{n, s, t}+\operatorname{res}_{n, t}+\text { dcinput }_{n, t}+\text { acinput }_{n, t}-\text { Demand }_{n, t} \quad \forall n, t \\
& g_{n, s, t} \leq \operatorname{Gmax}_{n, s} \\
& \forall n, s, t \\
& \operatorname{res}_{n, t} \leq \operatorname{Resmax}_{n, t} \\
& \text { dcinput }_{n, t}=\sum_{d} d c \text { flow }_{d, t} * \text { DCInc } c_{d, n} \\
& \text { acinput }_{n, t}=\sum_{n n}\left(B_{n, n n} * \delta_{n n, t}\right) \\
& \sum_{n} H_{l, n} * \delta_{n, t} \leq P F 0_{l}+P F \exp _{l} * \exp _{l}+P F u p_{l} * u p_{l} \\
& \sum_{n} H_{l, n} * \delta_{n, t} \geq-P F L 0-P F \exp _{l} * \exp _{l}-P F u p_{l} * u p_{l} \\
& d c \text { flow }_{d, t} \leq P F d c 0_{d}+\operatorname{expdc}_{d} * P F d c e x p_{d} \\
& d c f l o w_{d, t} \geq-P F d c 0_{d}-\operatorname{expdc}_{d} * P F d c e x p_{d} \\
& 0=\delta_{n n, t} * \text { Slack }_{n}
\end{aligned}
$$

The total system costs include the variable system cost of operation and the infrastructure cost of network investments. The applied methodology does not include combined investments in generation and transmission as the generation capacities are exogenous parameters provided by the EMF28 framework for the different scenarios.

The market dispatch determines the variable system cost of operation for a set of characteristic hours. It is constrained by:

- The nodal demand and available generation capacity for different technologies (2);

- Varying demand levels and availability of the conventional and renewable generation capacity for each hour (3) and (4);

- The network flow restriction by the transmission capacity of each link and the DC load flow constraint (5)- 10 .

In this setting, generation and inflows have to equal demand and outflows at all nodes in the network in every hour (2).

The main driver of infrastructure investment is the regional level of demand in relation to the spatial availability and cost of generation. It is not possible to operate the electricity system with the least cost generation capacities in case of network congestion as deviations from the merit order dispatch occur. Investments in new transmission 
links could relieve this congestion as additional exchange capacity is provided and the flow pattern in the meshed network changes. This could allow for a market dispatch with lower variable generation cost. An overall reduction in system cost is reached if the cost savings in the power plant dispatch are higher than the equivalent annuity for the transmission investment.

To converge to the cost minimal set of investments, the model has to be iterated several times. This is necessary due to the non-linear relation between the physical line characteristics, which change with investments and the voltage angle of the DC load flow approximation. The MILP endogenously assumes increasing line capacities with investments. The resulting changes in the flow pattern are included ex-post in the consecutive iteration as the parameters $B_{n, n n}$ and $H_{l, n}$ are recalculated after every iteration. Within the optimization process, investments that have been made in the same time step can be undone in the next iteration and the investment cost will be reimbursed. This approach results in convergence for the conducted model runs usually after less than ten iterations.

Each time step accounts for 10 years and for 2020, 2030, 2040, and 2050 the model optimizes the network topology in regard to the EMF28 scenarios thus applying a rolled planning approach, as the results of one calculation is the starting grid configuration for the consecutive time step. The size of the network and number of hours require a limitation of the binary and integer variables before optimizing for the entire year. This is done by reducing the model size into smaller sub-problems containing a limited set of hours. These smaller problems are solved before the full calculation and every upgrade and expansion which occurs in any of these runs remains in the solution space for the optimization for the full calculation.

The network topology consists of four non-synchronized high-voltage electricity grids $(150 \mathrm{kV}, 220 \mathrm{kV}, 300 \mathrm{kV}$, and $380 \mathrm{kV})$ that are connected by twelve high-voltage direct current (HVDC) cables. It has a total of 3,523 nodes (substations) and 5,145 lines as shown in Figure 1. This represents the transmission grid in all European countries synchronous with central Europe. Furthermore Scandinavia with Norway, Sweden, EastDenmark, Finland and the British Isles with the United Kingdom and Ireland. Each $\mathrm{AC}$ line is defined by the start and end node, its length, voltage level, and the number of installed circuits. The endogenous investment decisions include a binary decision for the voltage upgrade of lines to $380 \mathrm{kV}$ and an integer decision for $380 \mathrm{kV}$ lines to increase its number of circuits. 


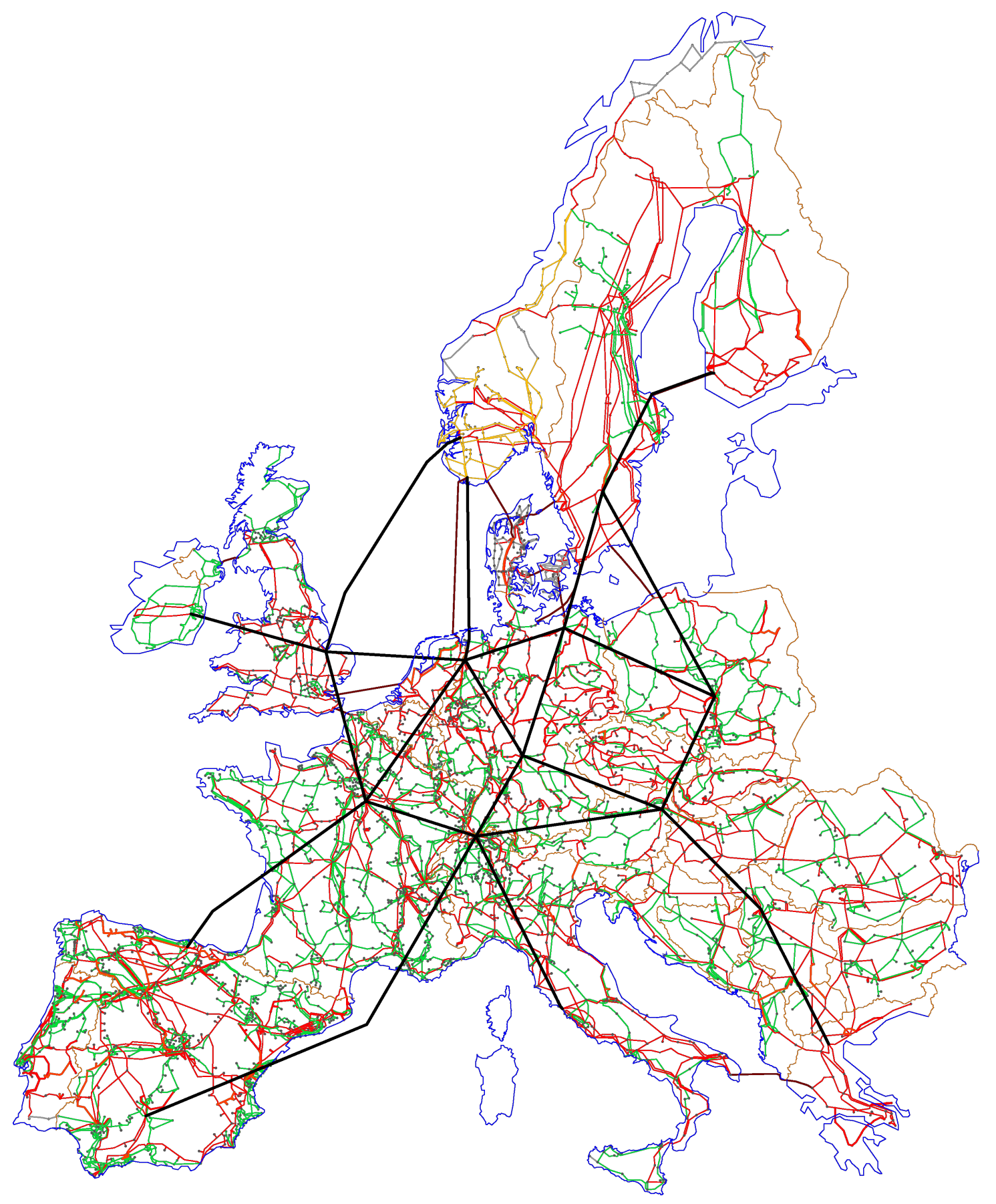

Figure 1: Initial Network Topology for Europe and DC Overlay Grid 
The DC lines are defined by a start and an end node, their capacity and length. The twelve existing DC lines are offshore connectors between the non-synchronized networks of Ireland, Great Britain, Scandinavia, and continental Europe. For the future network development, the model has the option to invest only in the lines of the overlay DC backbone grid consisting of 23 individual HVDC lines all over Europe.

\section{Data and Scenarios}

The infrastructure assessment is applied to the three EMF28 scenarios EU1, EU6 and EU10 using the results of the PRIMES model (Capros, 1998). The scenarios are distinguished by the two dimensions policy and technology: the policy measures define a certain mitigation level for greenhouse gas (GHG) emission which has to be reached by a limited set of technologies available, defined in the technology measures.

The EU1 scenario represents the reference policy scenario with the $20 \%$ reduction target for Europe by 2020 and a 40\% GHG reduction by 2050. The rest of the world (ROW) continues a "moderate policy". No emission trading scheme takes place on a global scale. No technology restrictions exist. CCS in fossil generation, nuclear power, renewable energy sources (RES) and energy efficiency follow a reference pathway leading to the $40 \%$ reduction of GHG.

The EU6 and EU10 scenario have more ambitious reduction targets for GHG. While the ROW remains with "moderate policy", Europe implements more progressive policies to reach a mitigation target of $80 \%$ by 2050 . Furthermore, the EU6 scenario sets no constraints on the use of nuclear and CCS, hence uses the same technology dimension as the EU1 scenario. In contrast the EU10 scenario constrains the usage of nuclear and CCS. PRIMES calculates a low share for nuclear and CCS. Assuming a higher level for energy efficiency, on the generation side most of the GHG reduction is reached by additional RES capacity.

The EU1 scenario serves as a reference scenario with no progressive policy implemented. The EU6 scenario allows for a comparison of the infrastructure needs assuming progressive policy with emissions reductions of $80 \%$ for GHG. The EU10 scenario assesses the impact on infrastructure if the currently pending choice about future technologies trends leans more towards renewable generation technologies. 
Investment costs are calculated for each individual line with regard to the technology and the type of investment (Table 1). It includes fixed investment costs for the transformer stations and variable costs for every kilometer of the line.

Table 1: Cost Factors for Transmission Investment

\begin{tabular}{ccc}
\hline Cost in $\mathrm{m} €$ & Transformer Stations & Line \\
\hline AC Expansion (per circuit) & 4.0 & 1.4 \\
AC Upgrade (per circuit) & 6.5 & 0.2 \\
DC Line (2GW circuit) & 260 & 1.4 \\
\hline
\end{tabular}

The top-down models calculate their data on a national level for the different scenarios. Based on data provided by PRIMES (generation capacity, annual demand, annual renewable generation output, resource prices for gas and coal, and the $\mathrm{CO}_{2}$ emission price) the input data for the infrastructure model is derived. Figure 2 shows the aggregated generation capacities for all countries to indicate the differences between the scenarios 5

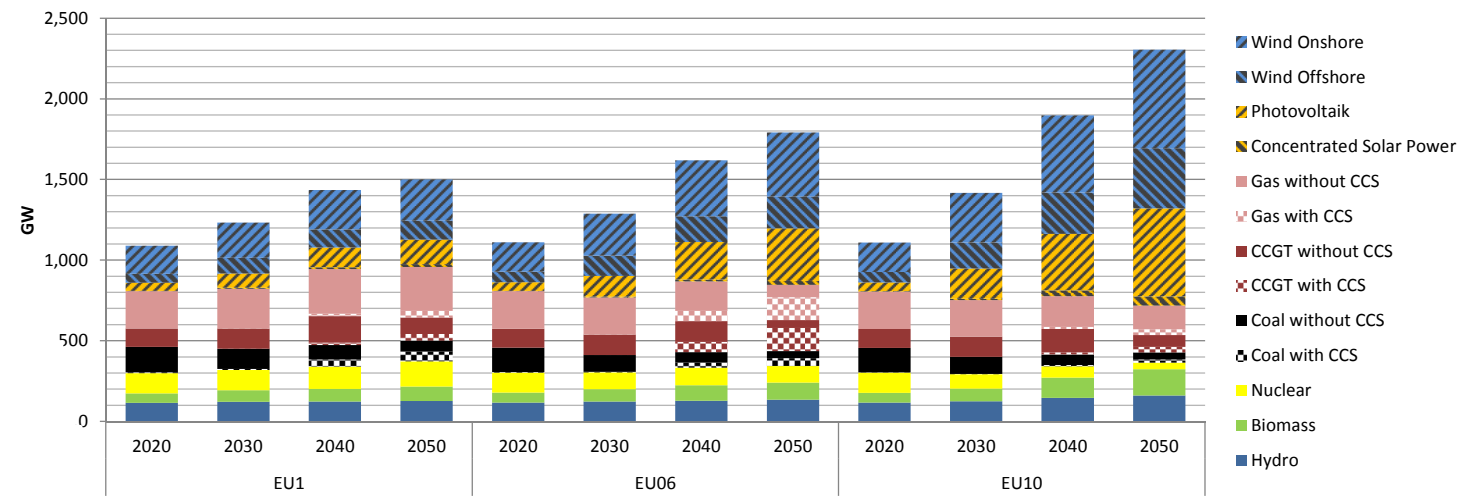

Figure 2: Aggregation of the Generation Capacities in the Scenarios for Europe. Source: PRIMES

The spatial character of the model requires nodal market data for generation and demand. Conventional generation capacity is distributed using geocoded data from the PLATTS power plant database (Platts, 2011) assuming a brown field approach for generation investment. These nodal capacities are scaled by scenario and year to fit the PRIMES data. The geographical information of the location of power plants is also used for its allocation to the nodes in the transmission network.

\footnotetext{
${ }^{5}$ The results of the PRIMES model cannot be stated on national level. The data section is limited to an aggregated overview for key scenario data on European level and the description of the regionalization of the national PRIMES data.
} 
The spatial allocation of the national PRIMES data for renewable generation uses a combination of the technical potential and the size of the NUTS 2 zones. The technical potential is deducted from the ReRiskreport by ESPON (2009). For onshore-wind, average wind speeds in $\mathrm{m} / \mathrm{s}$ are provided on a NUTS 2 level. For CSP and PV, the average radiation is provided as the $\mathrm{kWh}$ per year output by a $1 \mathrm{kWp}$ system mounted at optimum angle, also on a NUTS 2 level. The zonal potential is divided by the average potential in the country and then multiplied with the size of the NUTS 2 zone divided by the country size. This share is then allocated evenly to all nodes within the corresponding NUTS 2 zone. If no technical potential was available a combination of GDP and population factors was used.

To account for the fluctuating characteristics of feed-in of RES and demand fluctuations 18 representative hours were generated. These 18 hours consist of two seasons (summer, winter), three times of day (day, night, shoulder hours) and three wind availability cases (high, medium, low) as shown in Table 2 .

Table 2: Reference Hours

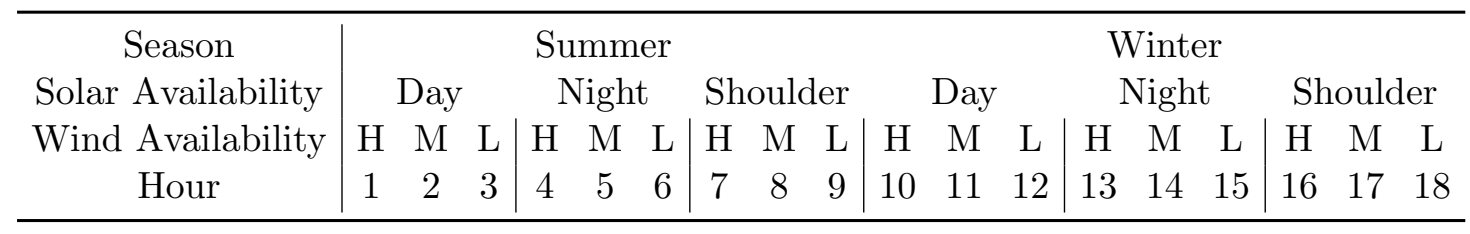

For photovoltaics (PV) and concentrated solar power (CSP) 70\% of the total electricity is generated in the summer and $30 \%$ during winter. The share of the energy generated over the course of day is shown in Table 3. In summer the amount of energy created

Table 3: Solar production energy share

\begin{tabular}{ccccccc}
\hline & \multicolumn{3}{c}{ Summer } & \multicolumn{3}{c}{ Winter } \\
\hline & Day & Night & Shoulder & Day & Night & Shoulder \\
PV & $71 \%$ & $0 \%$ & $29 \%$ & $83 \%$ & $0 \%$ & $17 \%$ \\
CSP & $71 \%$ & $0 \%$ & $29 \%$ & $83 \%$ & $0 \%$ & $17 \%$ \\
\hline
\end{tabular}

during the day vs. the evening is lower because days are longer; in winter most of the energy is produced during the day as days are shorter. For on- and offshore wind these shares are different for each country and provided by EC $(2012)$. The allocation between night, shoulder hours, day and low, medium, high can be found in Table 4 . 
Table 4: Wind production energy share

\begin{tabular}{lcccccc}
\hline & \multicolumn{3}{c}{ Summer } & \multicolumn{3}{c}{ Winter } \\
\hline & High & Mid & Low & High & Mid & Low \\
Onshore & $70 \%$ & $25 \%$ & $5 \%$ & $65 \%$ & $25 \%$ & $10 \%$ \\
Offshore & $60 \%$ & $30 \%$ & $10 \%$ & $55 \%$ & $30 \%$ & $15 \%$ \\
\hline
\end{tabular}

The yearly national demand has been spatially distributed to the different nodes based on the population of each node's corresponding NUTS 2 zone $(\mathrm{EC}, 2012)$. The demand is temporally differentiated for summer as well as winter and for shoulder hours, night and day. These shares result from an hourly aggregation of national demand from data taken from ENTSO-E (2012b).

As the PRIMES output is only reported for the EU27 countries, the installed capacity provided in the Platts-Database is used for non-EU27 countries. No change in the installed generation capacity over time is assumed. For Switzerland existing nuclear generation capacity is assumed to be decommissioned until 2040 and replaced by combined cycle gas power plants. Furthermore the installed hydro capacity in Norway is increased by $10 \%$ until 2050. For average and hourly availabilities the average of all EU27 countries is used.

\section{Results}

The results of the calculations for the three scenarios provide interesting insights in the grid expansion that is needed to provide an efficient dispatch while achieving minimal total cost.

The EU6 and EU10 scenarios target an 80\% GHG reduction until 2050 which imposes a significantly different generation mix that leads to more transmission investments. Furthermore the EU10 being a "green" scenario with the highest increase in RES is expected to have the highest expansion cost of all scenarios.

Table 5 shows the total investment costs over time for each scenario. The total overall investment both in terms of cost and kilometers in the scenario EU1 is lowest with $€ 31$ bn compared to the EU6 and EU10 scenarios with about $€ 57$ bn. The results are in the range of the Roadmap 2050 (EC, 2011) which assumes network investment costs between $€ 30$ bn and $€ 93 \mathrm{bn}$. The total investment cost for EU6 and EU10 do not vary 
significantly. Therefore, the difference between the "default" and the "green" scenario is not directly evident.

Table 5: Total investment costs for transmission capacity

\begin{tabular}{cccrrr}
\hline in $\mathrm{mn} \in$ & 2020 & 2030 & \multicolumn{1}{c}{2040} & \multicolumn{1}{c}{2050} & Total \\
\hline EU1 & 17,025 & 2,002 & 4,318 & 7,250 & 30,595 \\
EU6 & 18,864 & 4,318 & 18,670 & 15,067 & 56,919 \\
EU10 & 15,971 & 5,955 & 10,447 & 24,460 & 56,834 \\
\hline
\end{tabular}

The timing of investments reflect the scenario setting with the EU commitment to 2020 targets, no intermediate commitment to specific 2030 or 2040 targets and the 2050 target setting. For 2020 relatively high investment with little variance between the scenarios can be observed with an average of $€ 17 \mathrm{bn}$. For the subsequent years the investments vary; in 2030 the lowest amount is invested with an average of $€ 4 \mathrm{bn}$, as there are no strong reduction targets available for this time horizon. While the EU1 scenario remains on a low expansion path for transmission until 2050 the EU6 and EU10 scenario see high network investments after 2030 to reach the $80 \%$ reduction target. In the EU6 scenario more investments occur in 2040 compared to 2050 ( $€ 19$ bn versus $€ 15$ bn). The EU10 scenario has the larger share of investments in 2050 when RES levels reaching the $80 \%$ target.

Table 6: Total kilometers of upgrades or expansion

\begin{tabular}{cccrrr}
\hline in $\mathrm{km}$ & 2020 & 2030 & \multicolumn{1}{c}{2040} & \multicolumn{1}{c}{2050} & Total \\
\hline EU1 & 17,677 & 1,113 & 3,644 & 5,542 & 27,978 \\
EU6 & 18,664 & 2,914 & 18,288 & 12,556 & 52,424 \\
EU10 & 16,431 & 4,053 & 9,460 & 21,047 & 50,993 \\
\hline
\end{tabular}

Table 6 shows the sum of upgraded or expanded line kilometers. In the EU1 scenario the lowest number of lines is expanded resulting in the lowest total kilometers of the three scenarios. 2020 is the year with the highest investments, with an average of 17,500 km being expanded or upgraded. As all scenario assumptions for 2020 are similar the differences between the three scenarios is relatively small. The following time step 2030 shows the lowest expansion with an average of only $2,700 \mathrm{~km}$, corresponding with the lowest total investment cost in this year, but variations between scenarios begin to occur. While investments in the EU1 scenario in 2020 add up to only 1,100 km, 2,900 km and $4,000 \mathrm{~km}$ are upgraded in the EU6 and EU10 scenario respectively. This ratio 


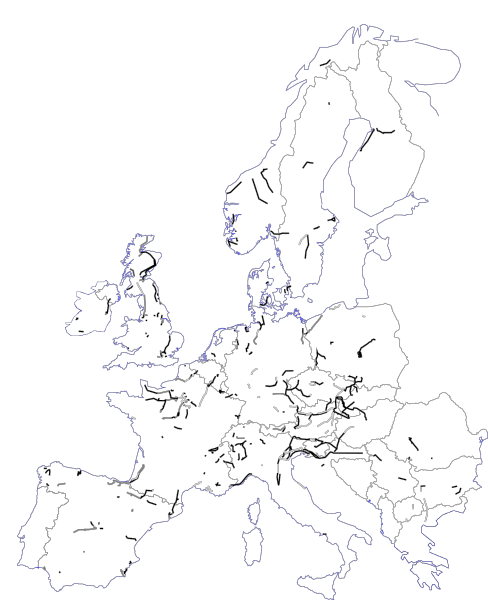

(a) EU1

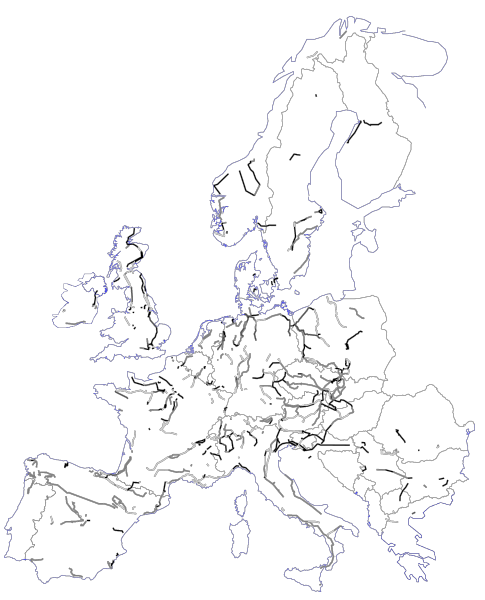

(b) EU6

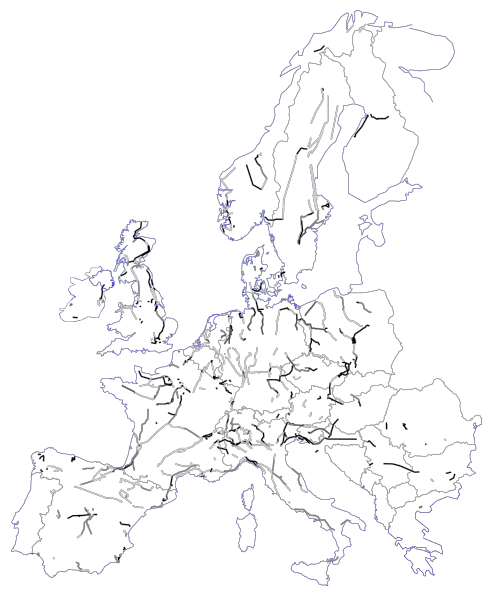

(c) EU10

Figure 3: AC grid infrastructure investments

remains relatively constant over all years resulting in almost twice as much kilometers in EU6 and EU10 compared to EU1 and shows that ambitious emission targets involve higher investments in grid infrastructure.

The investments in the AC grid are shown in Figure 3. In the scenario EU1 the grid investment need is about half that of the EU6 and EU10 scenarios. Here, mainly local grid reinforcement measures seem necessary. In the scenarios EU6 and EU10 more long-distance transmission is required. Especially in the scenario EU10 the increased north-south expansion structure indicates demand for long-distance transmission.

The results presented in Figure 4 indicate that only few HVDC lines are built. Most of the connectors are offshore. Thus HVDC lines are not built as an backbone in addition to the AC network but are integrated to connect the separated systems. The earlier an investment takes place the darker it is drawn.

Differences in national or cross-border grid expansion give insights in the long-range transmission capacity needs a scenario imposes on the system (Table 7). The EU1 scenario shows the lowest expanded km for DC and AC National. Both high-mitigation scenarios have comparable total $\mathrm{km}$ expansions and similar investments in national infrastructure but differ in the distribution on cross-border line types.

The EU6 scenario shows high demand for AC cross-border lines. This is not the case in the EU10 scenario where the investments in the long range DC grid infrastructure are significantly higher than the other scenarios. Figure 4 shows that the investments in 


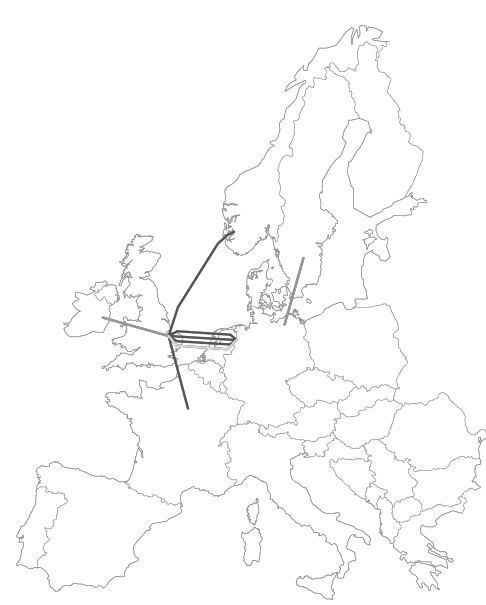

(a) EU1

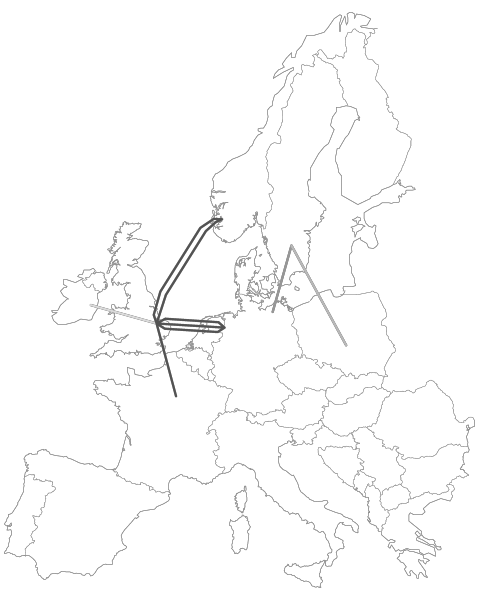

(b) EU6

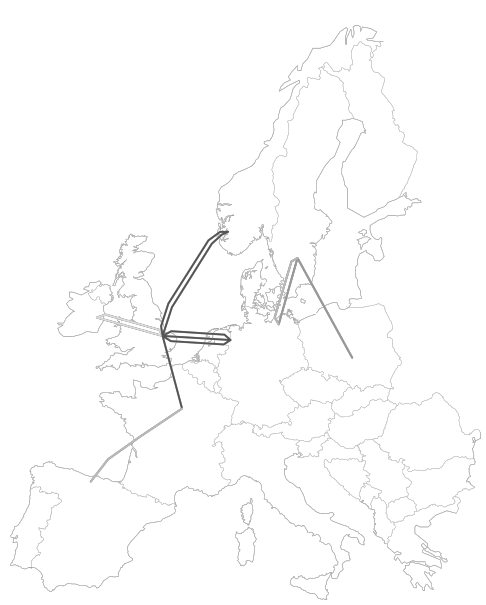

(c) EU10

Figure 4: DC grid infrastructure investments

Table 7: Kilometers per line type

\begin{tabular}{ccccc}
\hline in $\mathrm{mn} €$ & DC & AC National & AC Cross-Border & Total \\
\hline EU1 & 4,174 & 19,194 & 4,611 & 27,978 \\
EU6 & 5,346 & 39,905 & 7,173 & 52,424 \\
EU10 & 7,057 & 39,798 & 4,138 & 50,993 \\
\hline
\end{tabular}

the EU10 scenario connect into southern Europe to the Iberian Peninsula while in the other scenarios DC investments mainly function as means to connect countries separated by either the North or Baltic Sea. This indicates that with significant long distance transportation needs, the dominant AC expansion will be accompanied by complemental DC transmission capacity.

\section{Conclusion}

In this paper we explore model-based development scenarios for the future European electricity grid. Our point of inception are three scenarios for European electricity generation capacity, that differ i) by the degree of $\mathrm{CO}_{2}$-reduction ( $40 \%$ and $80 \%$ compared to 1990, respectively); and ii) by the share of renewables in the generation portfolio. We use a node- and line-sharp model of the European electricity market with a high granularity of techno-economic detail. We are particularly interested in the future architecture of the network, and the interplay between AC- and DC-development. In a 
European-wide analysis, we find that a strongly-meshed HVDC-network is unlikely to emerge. Note that this is in contrast with most of the literature, which assumes a future HVDC-meshed European grid. Instead, our model results suggest that the least cost solution includes investments in national $\mathrm{AC}$ networks and the expansion of $\mathrm{AC}$ interconnectors between countries. Thus a sensible expansion of the $380 \mathrm{kV} \mathrm{AC} \mathrm{grid} \mathrm{can} \mathrm{to}$ a certain degree substitute the development of a DC overlay grid. This is particularly visible in the EU10 scenario where only a single onshore DC connector between neighbouring countries is built despite high transmission demand. The different assumptions for each scenario have a significant influence on future transmission needs. Especially climate-oriented goals like GHG emission targets determine the grid capacities needed as renewable generation and demand are often geographically spread far apart. Comparing the scenario EU1 against EU6 and EU10 the resulting grid expansion in the low emission scenarios is almost twice as high both in terms of kilometers as well as investment cost.

\section{References}

Abrell, Jan and Kunz, Friedrich (2012): Integrating Intermittent Renewable Wind Generation - Insights from the Stochastic Electricity Market Model (stELMOD), TU Dresden Chair of Energy Economics. Working Paper.

Capros, Pantelis et al. (1998): The PRIMES energy system model-reference manual, National Technical University of Athens, Document as Peer Reviewed by the European Commission, Directorate General for Research.

EC (2006): Decision No 1364/2006/EC of the European Parliament and of the Council of 6 September 2006 laying down guidelines for trans-European energy networks and repealing Decision 96/391/EC and Decision No 1229/2003/EC.

EC (2011): Energy Roadmap 2050.

EC (2012): Eurostat Statistics Database.

Egerer, Jonas, Bückers, Lucas, and Drondorf, Gregor (2009): Sustainable energy networks for Europe - The integration of large-scale renewable energy sources until 2050, TU Dresden Chair of Energy Economics. Working Paper.

Egerer, Jonas, Kunz, Friedrich, and Von Hirschhausen, Christian (2012): Development scenarios for the North and Baltic Sea grid: A welfare economic analysis, DIW Berlin. Discussion Paper. 
ENTSO-E (2012a): 10-Year Network Development Plan 2012, Technical report, ENTSOE, Brussels.

ENTSO-E (2012b): ENTSO-E Consumption Data.

ESPON (2009): ReRisk - Regions at Risk of Energy Poverty, Technical report, ESPON, Luxembourg.

Leuthold, Florian, Jeske, Till, Weigt, Hannes, and Von Hirschhausen, Christian (2009): When the Wind Blows over Europe: A Simulation Analysis and the Impact of Grid Extensions, TU Dresden Chair of Energy Economics. Working Paper.

Leuthold, Florian, Weigt, Hannes, and von Hirschhausen, Christian (2012): A LargeScale Spatial Optimization Model of the European Electricity Market, Networks and Spatial Economics, 12(1):75-107.

Platts (2011): World Electric Power Plants Database.

Rosellón, Juan and Weigt, Hannes (2011): A dynamic incentive mechanism for transmission expansion in electricity networks: Theory, modeling, and application, The Energy Journal, 32(1):119-148.

Schill, Wolf-Peter, Rosellón, Juan, and Egerer, Jonas (2011): Regulated expansion of electricity transmission networks: The effects of fluctuating demand and wind generation, DIW Berlin. Discussion Paper.

Schweppe, Fred et al. (1988): Spot pricing of electricity, Kluwer Academic Publishers, Boston.

Weigt, Hannes and Hirschhausen, Christian von (2008): Price formation and market power in the German wholesale electricity market in 2006, Energy policy, 36(11):42274234 .

Weigt, Hannes, Jeske, Till, Leuthold, Florian, and von Hirschhausen, Christian (2010): Take the long way down: Integration of large-scale North Sea wind using HVDC transmission, Energy Policy, 38(7):3164-3173. 


\section{Appendix}

\section{Nomenclature}

\section{Sets}

$d \quad$ DC Lines in the electric grid

$l \quad$ AC Lines in the electric grid

$n$ Network node

$s \quad$ Power plant technology

$t$ Hour

\section{Parameters}

$B_{n, n n} \quad$ Network susceptance matrix

$C d c_{d} \quad$ DC Line expansion cost

Cexpl AC Line expansion cost per circuit

Cup $\quad$ AC Line upgrade cost

DCInc $c_{d, n} \quad$ DC line incidence matrix

Demand $_{n, t} \quad$ Electricity demand

$\operatorname{Gmax}_{n, s} \quad$ Max. Generation capacity

$H_{l, n} \quad$ Flow sensitivity matrix

$M C_{n, s} \quad$ Marginal production cost of generation

$\mathrm{PFO}_{l} \quad$ Initial AC power flow limit

$P F d c 0_{d} \quad$ Initial DC power flow capacity

$P F d c e x p_{d} \quad$ Additional DC capacity from expansion

$P F \exp _{l} \quad$ Additional AC capacity from expansion

$\mathrm{PFup}_{l} \quad$ Additional AC capacity from upgrade

$\operatorname{Resmax}_{n, t}$ Max. renewable generation capacity

Slack $_{n} \quad$ Slack bus

\section{Variables}

acinput $_{n, t}$ Input from the AC lines

$d_{c}$ flow $_{d, t} \quad$ Flow on dc lines

dcinput $_{n, t}$ Input from the DC lines

$\delta_{n, t} \quad$ Phase angle

expdc $\quad$ Expansion of DC line

$\exp _{l} \quad$ Expansion of AC line

$g_{n, s, t} \quad$ Generation of power plants

$r e s_{n, t} \quad$ Generation from renewable resources

$u p_{l} \quad$ Upgrade of AC line 